\title{
Correction: Uncertainty and depressive symptoms as mediators of quality of life in patients with heart failure
}

\section{Ting-Yu Chen, Chi-Wen Kao, Shu-Meng Cheng, Yue-Cune Chang}

Authors Ting-Yu Chen and Chi-Wen Kao are incorrectly noted as equal contributors to this work. The full, correct byline is as follows:

Ting-Yu Chen, Chi-Wen Kao*, Shu-Meng Cheng $\ddagger$ Yue-Cune Chang $\ddagger$.

$\ddagger$ These authors contributed equally to this work

* Corresponding author

\section{Reference}

1. Chen T-Y, Kao C-W, Cheng S-M, Chang Y-C (2018) Uncertainty and depressive symptoms as mediators of quality of life in patients with heart failure. PLoS ONE 13(11): e0205953. https://doi.org/10.1371/ journal.pone.0205953 PMID: 30427855

\section{G openaccess}

Citation: Chen T-Y, Kao C-W, Cheng S-M, Chang Y-C (2019) Correction: Uncertainty and depressive symptoms as mediators of quality of life in patients with heart failure. PLOS ONE 14(3): e0214825. https://doi.org/10.1371/journal.pone.0214825

Published: March 28, 2019

Copyright: ๑ 2019 Chen et al. This is an open access article distributed under the terms of the Creative Commons Attribution License, which permits unrestricted use, distribution, and reproduction in any medium, provided the original author and source are credited. 